\title{
Efficacy of Percutaneous Transforaminal Endoscopic Discectomy for the Treatment of Patients Undergoing Lumbar Disc Herniation With Positive Nerve Root Sedimentation Sign: a Retrospective Case-Control Study
}

\section{Luoyi Deng}

Guizhou Medical University

Hua Yang

Guizhou Medical University

Miao Liu

Guizhou Medical University

Jin Deng

Guizhou Medical University

Guoxuan Peng

Guizhou Medical University

Xu Ning

Guizhou Medical University

Hong Sun ( $\sim$ sunhong002@12.com )

Guizhou Medical University https://orcid.org/0000-0002-4195-501X

Research article

Keywords: Lumbar disc herniation, percutaneous transforaminal endoscopic discectomy, Nerve root sedimentation sign

Posted Date: July 24th, 2020

DOI: https://doi.org/10.21203/rs.3.rs-38294/v1

License: (c) (1) This work is licensed under a Creative Commons Attribution 4.0 International License. Read Full License 


\section{Abstract}

Background: To investigate the efficacy of percutaneous transforaminal endoscopic discectomy (PTED) in the treatment of patients who were diagnosed with lumbar disc herniation (LDH) with positive nerve root sedimentation sign (NRSS).

Methods: A total of 86 patients who underwent LDH were recruited and divided into NRSS-positive group $(n=49)$ and NRSS-negative group $(n=37)$. The visual analog scale (VAS), Japanese Orthopaedic Association (JOA), and Oswestry Disability Index (ODI) were used to evaluate the low back pain and functional recovery and were compared between the two groups.

Results: There were no significant differences in the demographic parameters between the two groups. The average area of the dural sac compression in the NRSS-positive group was significantly higher than that in the NRSS-negative group. Patients with a positive NRSS showed a better low back pain relief than those with a negative NRSS at 1 week and 1 month after surgery. The JOA and ODI in the NRSS-positive group were better than those in the NRSS-negative group at 3 months and 6 months postoperatively. The apparent efficiency of JOA and the excellent and good rate of ODI in the negative group was lower than that in the positive group at 6 months after surgery.

Conclusions: Patients undergoing LDH with a positive NRSS showed better pain relief and functional recovery than those with a negative NRSS. The present study suggested that NRSS might be a valuable sign and associated with better clinical outcomes in patients undergoing LDH with the treatment of PTED.

\section{Background}

Epidemiological investigations show that LDH is a common cause of low back pain [1]. The minimally invasive technique for the treatment of LDH is percutaneous transforaminal endoscopic discectomy (PTED) [2]. Compared with traditional surgery, this technique has the advantages of less trauma and pain and lower infection rate, which is beneficial for early rehabilitation and functional recovery [3]. While minimally invasive techniques continue to evolve, imaging diagnostic techniques for lumbar disease are also being enriched. In 2010, Barz et al. [4] proposed that the NRSS shows high sensitivity and specificity in the diagnosis of lumbar spinal stenosis. Therefore, it can be used as a new imaging index to assist the diagnosis of lumbar spinal stenosis [5-7]. Patients with a positive NRSS suffer from poorer clinical outcomes than those with a negative NRSS when they are undergoing conservative therapies [8]. Interestingly, patients with a positive NRSS have better clinical outcomes than those with a negative NRSS when they are undergoing surgeries $[9,10]$. However, no study has investigated the correlation between NRSS and postoperative outcomes in patients with LDH. In the present study, we aimed to investigate the clinical efficacy of patients with LDH undergoing PTED and explore the association between clinical outcomes and NRSS. 


\section{Methods}

\section{Study design and patient's recruitment}

This study retrospectively analyzed patients with LDH admitted from January 2017 to September 2018 in the Affiliated Hospital of Guizhou Medical University. The nature of nerve root sedimentation sign was determined according to the lumbar MRI cross-section. Inclusion criteria were the central or paracentral type of LDH in a single-segment, and the clinical symptoms consistent with the imaging lesions. The patients treated conservatively for 3 months and the symptoms did not improve. Exclusion criteria were patients with lumbar spinal stenosis, LDH in L5/S1 segment, spinal fractures, spondylolisthesis, malignant tumors, and lateral deformities or with severe hip or knee osteoarthritis. This study was approved by the ethics committee of the Affiliated Hospital of Guizhou Medical University.

\section{Evaluation of NRSS and measurement of the compressed percentage of dural sac}

The lumbar spine axial T2-weighted MR images were obtained with a GE HD Signa 3.0T MR Scanner. Using the method described by Barz et al [4], we made a straight line between the apex of the small joints on both sides of the lumbar spine and divided the lumbar spine into 2 parts (Fig. 1a and b). If the nerve roots were settled to the dorsal side of the dural sac due to gravity, it was called negative NRSS (Fig. 1c). Conversely, if the nerve roots were scattered and floated in the center of the dural sac, it was called positive NRSS (Fig. 1d). In addition, except for the L5/S1 segment, whether in the plane of the disc herniation or in the vicinity of the plane, as long as there was a positive sign at any MRI image level, the patient was defined as a NRSS-positive patient. Each lumbar intervertebral disc was scanned for three layers. By using a Picture Archiving and Communication System (PACS) with integrated digital area measurement, the cross-sectional areas of the dural sac (Fig. 2b) and herniated disc were measured (Fig. 2c), and thus, the compressed percentage of the dural sac (S2/S1) was calculated. The maximum area ratio among the three layers was selected as the compressed percentage of the dural sac. The results were evaluated by two investigators independently (Luoyi Deng and Hua yang). When the difference was encountered, the third investigator (Hong Sun) helped to reach a consensus with all investigators.

\section{Outcomes measurement}

Baseline data, operation time, hospital stay, and postoperative complications were recorded. The VAS score was used to assess the preoperative and postoperative pain. The JOA and ODI score were used to evaluate the low back pain and functional improvement. Macnab score was adopted to evaluate the clinical efficacy.

\section{Statistical methods}

All statistical analyses were performed using SPSS version 20.0 (SPSS Inc, Chicago, USA). After compliance with normality test and confirmation,continuous variables were presented as mean \pm 
standard deviations, whereas classified variables were expressed as percentages. Independent sample $t$ test or one-way ANOVA was used for continuous variables, while chi-square test or Fisher's exact test was used for counting data. $P$ values of less than 0.05 were considered to be statistically significant.

\section{Results}

\section{General information}

A total of 86 patients were enrolled in the present study and divided into NRSS-positive $(n=49)$ and negative groups $(n=37)$. Demographic characteristics of all patients are presented in Table 1 . There were 21 males and 16 females in NRSS-negative group, with an average age of $50.27 \pm 13.25$ years and an average body mass index (BMI) of $23.77 \pm 3.38 \mathrm{~kg} / \mathrm{m}^{2}$. In NRSS-positive group, there were 27 males and 22 females with an average age of $53.29 \pm 15.05$ years old and an average BMI of $23.34 \pm 3.49 \mathrm{~kg} / \mathrm{m}^{2}$. No significant differences in demographic parameters including gender, age, and BMI were found between the two groups. Moreover, no significant differences were found between groups with respect to the duration of disease, comorbidity, operation time, and length of hospital stay.

\section{The incidence of positive NRSS in the different type of $L D H$}

Radiographic characteristics between the two group are available in Table 2. LDH can be divided into central and paracentral types. There was a total of 31 patients with central LDH and 55 patients with paracentral LDH. The compressed percentage of dural sac in patients with central LDH was greater than that in patients with paracentral LDH $((31 \pm 6) \%$ versus $(21 \pm 5) \%, t=9.211, P<0.001)$. In addition, the compressed percentage of the dural sac between the groups showed significant differences. There were 4 patients with central type and 33 with paracentral type in the NRSS-negative group. However, there were 27 patients with central LDH and 22 patients with paracentral LDH in the NRSS-positive group. Compared to the patients with paracentral $L D H$, the patients with central type of LDH were more likely to get positive NRSS.

\section{The incidence of positive NRSS in different lumbar segments}

The incidence of positive NRSS in all patients was $56.98 \%$ (49/86). Further, analysis showed that the incidence of positive NRSS in different lumbar segments was different. There were 2 cases $(2.33 \%)$ in L2/3 segment, 5 cases (5.81\%) in L3/4 segment and 42 cases (48.84\%) in L4/5 segment. The incidence of positive NRSS in L4/5 segment was significantly greater than that in L2/3 and L3/4 segments $\left(c^{2}=6.386, P=0.037\right)$.

The correlation between the incidence of positive NRSS and the compressed percentage of dural sac

The compressed percentage of dural sac in the present study ranged between 0 to $40 \%$. As shown in Table 2, the incidence of positive NRSS was about 28.20\% (11/39) when the compressed percentage of 
dural sac was less than $20 \%$. As for the compressed percentage of dural sac more than $20 \%$, the incidence of positive NRSS was about $80.85 \%$ (38/47).

\section{Clinical outcomes}

As shown in Table 3, the preoperative clinical outcomes were evaluated by using VAS, JOA and ODI scores. The results showed that all the patients who underwent PTED had good clinical outcomes in pain relief and functional recovery. Moreover, the patients with positive NRSS had a better pain relief than those with negative NRSS, at 1 week and 1 month after surgery. However, there were no significant differences at 3 and 6 months after surgery. In terms of JOA and ODI scores, the patients with positive NRSS had a better functional recovery than those with negative NRSS at 3 and 6 months after surgery. However, no significant differences were found at 1 month after surgery between the groups.

The apparent rate of JOA scores at 6 months after surgery was $67.57 \%$ (Table 4 ) in the negative group and $85.71 \%$ in the positive group. The superior rate of ODI scores in the two groups at 6 months after surgery were $67.57 \%$ and $87.76 \%$, respectively. These differences between groups were statistically significant (Table 5). The excellent and good rates of Macnab scores in the two groups at 6 months after surgery were $81.08 \%$ and $89.80 \%$, respectively. There was no significant difference in Macnab scores between groups at 6 months after surgery (Table 6). In addition, there were no patients with complications including nerve root injury, intraspinal infection and dural sac rupture in both groups.

\section{Discussion}

Numerous studies have investigated the prognostic roles of NRSS with spinal stenosis [4,5]. However, whether this sign can be used for the evaluation of LDH treatment is still unknown. Our results showed that NRSS may be a valuable sign and associated with clinical outcomes in patients with LDH who undergo PTED. NRSS can be found in the patients with LDH and the positive rate of NRSS is about $63.1 \%$ [11]. Our results showed that the positive rate was $56.98 \%$. Moreover, the positive NRSS mainly occurred in the L4/5 segment. It was reported that the nerve roots in the L5/S1 segment always float and thus remain positive. Therefore, the patients with LDH in the L5/S1 segment were not included in the present study.

It is reported that the pressure of the dural sac is closely related to the incidence of positive NRSS $[12,13]$. In the present study, our results suggested that the patients with central LDH had higher positive rates than the patients with paracentral LDH. The epidural compression was different between central and paracentral type of LDH. The compressed percentage of dural sac in patients with central LDH was always greater than that in patients with paracentral LDH. In addition, the patients appeared to be more susceptible to get the positive NRSS when the compressed percentage of dural sac was more than $20 \%$. Hence, our results also indicated that the degree of compressed dural disc was positively associated with the occurrence of positive NRSS. In addition, we noted that several patients with little pressure of the dural sac suffered from the positive NRSS, suggesting that compression of the dural sac might not be the only factor leading to the occurrence of positive NRSS. 
The correlation between NRSS and clinical outcomes was also explored in the current study. All included subjects were with single-segment LDH with the aim to avoid the influence on the evaluation of NRSS from adjacent segments. Our results showed the patients with a positive NRSS had a decreased VAS scores at 1 week and 1 month after surgery and ODI scores at 3 and 6 months after surgery. Moreover, the patients with positive NRSS had a better JOA scores than those with negative NRSS at 3 and 6 months after surgery. Furthermore, the apparent efficiency of JOA scores and the excellent rates of ODI scores in patients with positive NRSS were improved compared to the patients with negative NRSS. These findings indicated that the patients with positive NRSS might have better clinical outcomes than those with negative NRSS. The NRSS may act as a valuable sign for the postoperative outcomes for the patients with LDH undergoing PTED. The VAS scores in positive group were decreased than that in negative group at 3 and 6 months after surgery. However, no significant difference was found between the groups. Both groups showed similar effects with respect to JOA and ODI sores at 1 month after surgery. These findings may be attributed to several factors. The VAS score is mainly based on the subjective experiences of patient's pain level. Generally, the nerve roots were more compressed in positive group than that in negative group. Hence, the pain levels of lower limbs were significantly decreased in positive group at 1 week and 1 month after the physical compression of nerve roots was removed. The edema and inflammation of nerve roots would be eliminated at 3 months after surgery. Therefore, there were no significant findings between groups in VAS scores. The JOA and ODI scores include not only subjective experiences of patients' pain level but also clinical outcomes such as daily life improvement and lower limbs recovery. Although the compression of nerve roots was removed, it would take some time for the improvement of clinical outcomes. Hence, we could not find any significant difference in JOA and ODI scores between groups at 1 month after surgery.

It is showed that the patients with positive NRSS have higher improvement rates than the patients with negative NRSS in both JOA and ODI scores at 6 months after surgery. Several reasons may be attributed to these. Firstly, the patients with positive NRSS always suffer from higher compressed pressure of the dural sacs and nerve roots than those with negative NRSS. Secondly, more inflammatory cytokines may be released from the lumbar intervertebral discs in positive group. In addition, our results failed to show any statistical difference in Macnab scores between the groups. These findings might be because Macnab score is mainly based on the femoral nerve stretch test, which will be more significant for the LDH in L2/3 and L3/4 segments. Most patients in the present study were with LDH in L4/5 segment, which might attribute to the non-statistical findings in the excellent rate of Macnab scores between groups at half year after surgery.

Nevertheless, several limitations should be cautioned in the present study. Firstly, some studies have investigated the transitions from NRSS positive to negative after surgery [14]. Most patients in the present study were reluctant to return to hospital for MRI examination as the pain was relieved by PTED surgery. Hence, we were unable to explore the transition of NRSS with insufficient data. Secondly, the long-term difference between groups was not investigated due to the short follow-up. Thirdly, only 86 patients were enrolled in our study. Therefore, such findings should be further confirmed by larger sample size and multi-center studies. 


\section{Conclusions}

The current study indicated that patients undergoing LDH with positive NRSS showed better pain relief and functional recovery when compared to those with negative NRSS. The present study suggested that NRSS might be a valuable sign and associated with clinical outcomes in patients undergoing LDH with the treatment of PTED.

\section{Declarations}

\section{Ethics approval and consent to participate}

This study was approved by the ethics committee of the Affiliated Hospital of Guizhou Medical University. Informed written consent was obtained from all patients.

\section{Consent for publication}

The publication of present manuscript was reached consensus by all authors.

\section{Availability of data and materials}

All data are fully available without restriction.

\section{Competing interests}

All authors declare that there is no conflict of interests.

\section{Abbreviations}

PTED, percutaneous transforaminal endoscopic discectomy. LDH, lumbar disc herniation. NRSS, nerve root sedimentation sign. VAS, visual analog scale. JOA, Japanese Orthopaedic Association. ODI, Oswestry Disability Index. PACS, Picture Archiving and Communication System.

\section{Funding}

This work was supported by the science and technology funds from Guizhou Provincial Health Commission (Grant NO. gzwjkj2019-1-134).

\section{Acknowledgments}

The authors thank Prof. Chuan Ye and Xianwen Shang at the Affiliated Hospital of Guizhou Medical University for assistance with writing. The authors would also like to express their gratitude to EditSprings (https://www.editsprings.com/) for the expert linguistic services provided.

\section{References}


1. Ambrossi GL, McGirt MJ, Sciubba DM, Witham TF, Wolinsky JP, Gokaslan ZL, Long DM (2009) Recurrent lumbar disc herniation after single-level lumbar discectomy: Incidence and health care cost analysis. Neurosurgery 65 (3):574-578. doi:10.1227/01.Neu.0000350224.36213.F9

2. Wang K, Hong X, Zhou BY, Bao JP, Xie XH, Wang F, Wu XT (2015) Evaluation of transforaminal endoscopic lumbar discectomy in the treatment of lumbar disc herniation. International orthopaedics 39 (8):1599-1604. doi:10.1007/s00264-015-2747-1

3. Nellensteijn J, Ostelo R, Bartels R, Peul W, van Royen B, van Tulder M (2010) Transforaminal endoscopic surgery for symptomatic lumbar disc herniations: A systematic review of the literature. Eur Spine J 19 (2):181-204. doi:10.1007/s00586-009-1155-x

4. Barz T, Melloh M, Staub LP, Lord SJ, Lange J, Roder CP, Theis JC, Merk HR (2010) Nerve root sedimentation sign: evaluation of a new radiological sign in lumbar spinal stenosis. Spine (Phila Pa 1976) 35 (8):892-897. doi: 10.1097/BRS.0b013e3181c7cf4b

5. Macedo LG, Wang Y, Battie MC (2013) The sedimentation sign for differential diagnosis of lumbar spinal stenosis. Spine (Phila Pa 1976) 38 (10):827-831. doi: 10.1097/BRS.0b013e31827e8ecd

6. Wang G, Peng Z, Li J, Song Z, Wang P (2019) Diagnostic performance of the nerve root sedimentation sign in lumbar spinal stenosis: a systematic review and meta-analysis. Neuroradiology 61 (10):1111-1121. doi:10.1007/s00234-019-02248-3

7. Zhang L, Chen R, Liu B, Zhang W, Zhu Y, Rong L (2017) The nerve root sedimentation sign for differential diagnosis of lumbar spinal stenosis: a retrospective, consecutive cohort study. Eur Spine J 26 (10):2512-2519. doi:10.1007/s00586-016-4435-2

8. Barz T, Staub LP, Melloh M, Hamann G, Lord SJ, Chatfield MD, Bossuyt PM, Lange J, Merk HR (2014) Clinical validity of the nerve root sedimentation sign in patients with suspected lumbar spinal stenosis. Spine J 14 (4):667-674. doi: 10.1016/j.spinee.2013.06.105

9. Moses RA, Zhao W, Staub LP, Melloh M, Barz T, Lurie JD (2015) Is the sedimentation sign associated with spinal stenosis surgical treatment effect in SPORT? Spine (Phila Pa 1976) 40 (3):129-136. doi:10.1097/BRS.0000000000000672

10. Zhang G, Gao X, Chen M, Wang D, Li J (2016) Correlation between the nerve root sedimentation and the lumbar disc herniation. Anhui Medical and Pharmaceutical Journal 20 (5):883-886. doi: 10.3969/j.issn.1009-6469.2016.05.018

11. Tian P, Fu X, Sun X, Deng S, Ma X (2014) The different sign of nerve root sedimentation on MRI in patients between lumbar spondylolisthesis and lumbar disc herniation. Tianjin Medical Journal (12):1216-1219. doi: 10.3969/j.issn.0253-9896.2014.12.018

12. Barz T, Melloh M, Staub LP, Lord SJ, Lange J, Merk HR (2014) Increased intraoperative epidural pressure in lumbar spinal stenosis patients with a positive nerve root sedimentation sign. Eur Spine $J$ 23 (5):985-990. doi:10.1007/s00586-013-3071-3

13. Fazal A, Yoo A, Bendo JA (2013) Does the presence of the nerve root sedimentation sign on MRI correlate with the operative level in patients undergoing posterior lumbar decompression for lumbar stenosis? Spine J 13 (8):837-842. doi: 10.1016/j.spinee.2013.02.067 
14. Barz C, Melloh M, Staub LP, Lord SJ, Merk HR, Barz T (2017) Reversibility of nerve root sedimentation sign in lumbar spinal stenosis patients after decompression surgery. Eur Spine J 26 (10):2573-2580. doi:10.1007/s00586-017-4962-5

\section{Tables}

Table 1: Demographic characteristics between the two groups

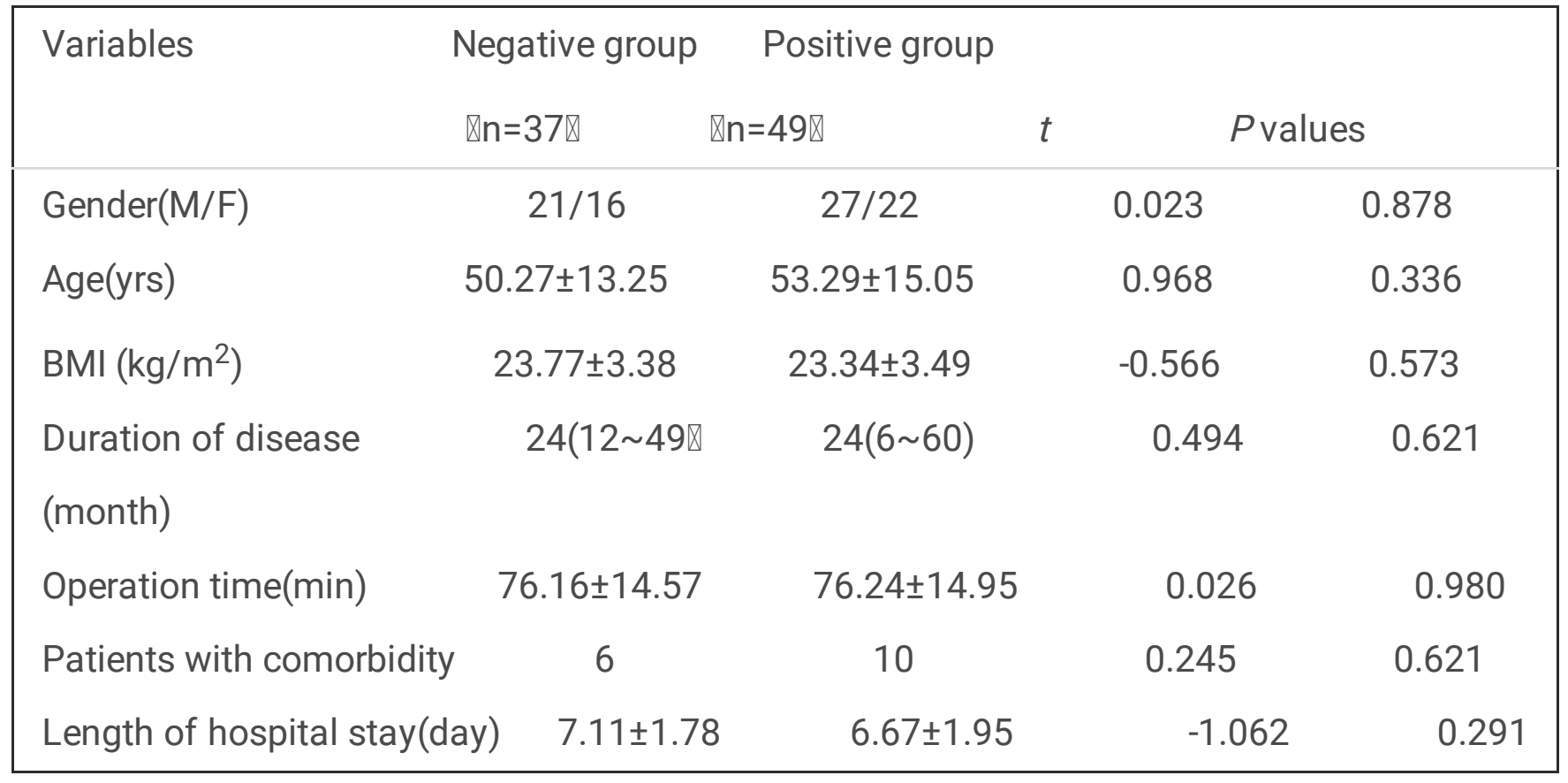

Table 2: Radiographic characteristics between the two group

\begin{tabular}{|lcccc|}
\hline Variables & $\begin{array}{c}\text { Negative group } \\
(\mathrm{n}=37)\end{array}$ & $\begin{array}{c}\text { Positive group } \\
(\mathrm{n}=49)\end{array}$ & $t / \mathrm{c}^{2}$ & $P$ values \\
$\begin{array}{l}\text { Compressed percentage } \\
\text { of dural sac (\%) }\end{array}$ & & & & \\
Average & $20 \pm 6$ & $28 \pm 6$ & 6.210 & 0.000 \\
$0-20 \%$ & 28 & 11 & & \\
$21-40 \%$ & 9 & 38 & 24.099 & 0.000 \\
$\begin{array}{l}\text { Type of LDH } \\
\text { Central type }(\mathrm{n}=31)\end{array}$ & $4(12.90 \%)$ & $27(87.10 \%)$ & & \\
Paracentral $(\mathrm{n}=55)$ & $33(60.00 \%)$ & $22(40.00 \%)$ & 17.939 & 0.000 \\
\hline
\end{tabular}


Table 3: Comparison of perioperative clinical outcomes between the two groups ( $\square x \pm s$ )

\begin{tabular}{|c|c|c|c|c|c|c|}
\hline & Group & Preoperative & $\begin{array}{l}\text { Postoperative } \\
1 \text { week }\end{array}$ & $\begin{array}{l}\text { Postoperative } \\
1 \text { month }\end{array}$ & $\begin{array}{l}\text { Postoperative } \\
3 \text { months }\end{array}$ & $\begin{array}{l}\text { Postoperative } \\
6 \text { months }\end{array}$ \\
\hline \multirow[t]{6}{*}{ VAS } & $\begin{array}{l}\text { Negative } \\
\text { group }\end{array}$ & $7.24 \pm 0.98$ & $3.14 \pm 0.59^{\#}$ & $2.84 \pm 0.60^{\#}$ & $2.46 \pm 0.61^{\#}$ & $1.62 \pm 0.76^{\#}$ \\
\hline & $(n=37)$ & & & & & \\
\hline & $\begin{array}{l}\text { Positive } \\
\text { group }\end{array}$ & $7.29 \pm 0.87$ & $2.82 \pm 0.53^{\#}$ & $2.55 \pm 0.6$ \# & $2.22 \pm 0.59^{\#}$ & $1.45 \pm 0.82^{\#}$ \\
\hline & $(n=49)$ & & & & & \\
\hline & $t$ & 0.212 & -2.648 & -2.163 & -1.814 & -1.000 \\
\hline & $P$ & 0.832 & 0.010 & 0.033 & 0.073 & 0.320 \\
\hline \multirow[t]{6}{*}{ JOA } & $\begin{array}{l}\text { Negative } \\
\text { group }\end{array}$ & $11.43 \pm 1.64$ & - & $19.92 \pm 1.3^{\#}$ & $23.51 \pm 1.69^{\#}$ & $24.38 \pm 1.99^{\#}$ \\
\hline & $(n=37)$ & & & & & \\
\hline & $\begin{array}{l}\text { Positive } \\
\text { group }\end{array}$ & $10.92 \pm 1.98$ & - & $19.98 \pm 1.28 \#$ & $24.82 \pm 1.87^{\#}$ & $25.22 \pm 1.62^{\#}$ \\
\hline & $(n=49)$ & & & & & \\
\hline & $t$ & -1.282 & - & 0.216 & 3.333 & 2.109 \\
\hline & $P$ & 0.203 & - & 0.830 & 0.001 & 0.039 \\
\hline \multirow[t]{6}{*}{ ODI } & $\begin{array}{l}\text { Negative } \\
\text { group }\end{array}$ & $52.70 \pm 8.40$ & - & $29.15 \pm 4.54^{\#}$ & $26.36 \pm 3.99^{\#}$ & $16.89 \pm 7.69^{\#}$ \\
\hline & $(n=37)$ & & & & & \\
\hline & $\begin{array}{l}\text { Positive } \\
\text { group }\end{array}$ & $53.87 \pm 8.15$ & - & $28.53 \pm 4.72^{\#}$ & $23.31 \pm 4.38^{\#}$ & $13.60 \pm 5.49^{\#}$ \\
\hline & $(n=49)$ & & & & & \\
\hline & $t$ & 0.649 & - & -0.610 & -3.319 & -2.31 \\
\hline & $P$ & 0.518 & - & 0.544 & 0.001 & 0.030 \\
\hline
\end{tabular}

Note: \#, compared with preoperative, $P<0.05$. 
Table 4: The postoperative improvement rate of JOA scores between the groups at 6 months

\begin{tabular}{|c|c|c|c|c|c|c|}
\hline \multirow[t]{2}{*}{ Group } & \multirow[t]{2}{*}{ Excellent } & \multirow[t]{2}{*}{ Valid } & \multirow[t]{2}{*}{ Invalid } & Apparent & \multirow[t]{2}{*}{ Effective } & \multirow[t]{2}{*}{$\mathrm{c}^{2} \quad P$ values } \\
\hline & & & & rate rate & & \\
\hline Negative group & 25 & 12 & 0 & $67.57 \%$ & $100 \%$ & \\
\hline$(n=37)$ & & & & & 4.033 & 0.045 \\
\hline $\begin{array}{l}\text { Positive group } \\
(n=49)\end{array}$ & 42 & 7 & 0 & $85.71 \%$ & $100 \%$ & \\
\hline
\end{tabular}

Note: the postoperative JOA improvement rate $=[$ (postoperative JOA score - preoperative JOA score) $/(29$ - preoperative JOA score)] $\times 100 \%$. Excellent, the improvement rate is between $61 \%$ and $100 \%$. Valid, the improvement rate is between $25 \%$ and $60 \%$. Invalid, the improvement rate is less than $25 \%$. Apparent rate $=($ number of excellent cases $/$ numbers of total cases $) \times 100 \%$. Effective rate $=[$ (number of total cases number of invalid cases) / number of total cases] $\times 100 \%$.

Table 5 The postoperative improvement rate of ODI scores between the groups at 6 months after surgery

\begin{tabular}{lcccccc}
\hline Group & $\begin{array}{c}\text { Improvement rate } \\
\geq 50 \%\end{array}$ & $\begin{array}{c}\text { Improvement rate } \\
\geq 25 \%\end{array}$ & Superior rate & Effective rate & $\chi^{2}$ & $P$ values \\
\hline $\begin{array}{c}\text { Negative group } \\
(\mathrm{n}=37)\end{array}$ & 25 & 37 & $67.57 \%$ & $100 \%$ & & \\
$\begin{array}{c}\text { Positive group } \\
(\mathrm{n}=49)\end{array}$ & 43 & 49 & & & 5.191 & 0.023 \\
& & & & & & \\
\hline
\end{tabular}

Note: the postoperative ODI improvement rate $=$ (preoperative ODI score - postoperative ODI score) / preoperative ODI score $\times 100 \%$. Superior rate $=$ number of cases with improvement rate $\geq 50 \% /$ number of total cases $\times 100 \%$. Effective rate $=$ number of cases with improvement rate $\geq 25 \% /$ total number of patients $\times 100 \%$. 
Table 6 the postoperative Macnab scores between two groups at 6 months after surgery

\begin{tabular}{lccccccc}
\hline Group & Excellent & Good & Middle & Poor & Superior rate & $\chi^{2}$ & $P$ values \\
\hline $\begin{array}{c}\text { Negative group } \\
\text { (n=37) }\end{array}$ & 20 & 10 & 7 & 0 & $81.08 \%$ & & \\
$\begin{array}{c}\text { Positive group } \\
(\mathrm{n}=49)\end{array}$ & 35 & 9 & 5 & 0 & $89.80 \%$ & & \\
\hline
\end{tabular}

Note: Superior rate $=($ number of cases with excellent and good Macnab scores $/$ number of total cases $\times 100 \%$

\section{Figures}




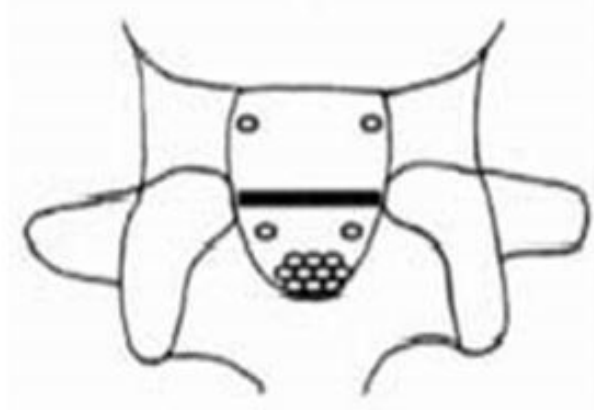

a negative sedimentation sign

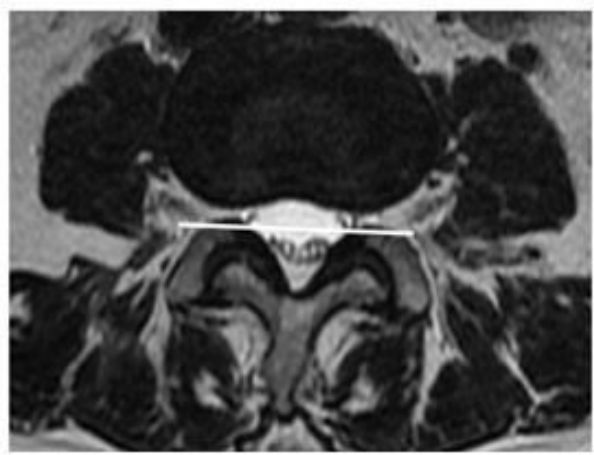

c the diagram of negative nerve root sedimentation sign in MRI. The nerve roots were settled to the dorsal side of the dural sac.

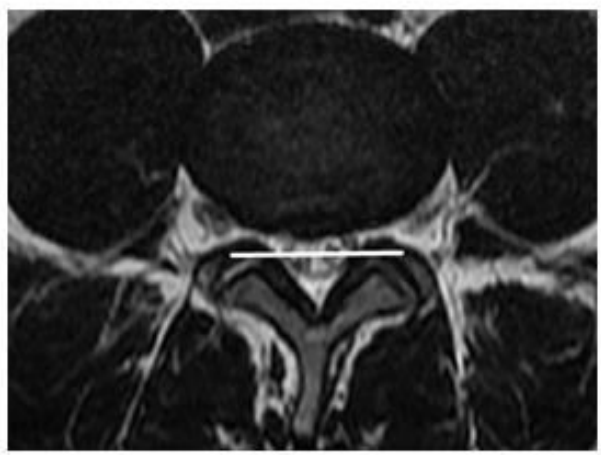

$\mathrm{d}$ the diagram of positive nerve root sedimentation sign in MRI. The nerve roots were scattered and floated in the center of the dural sac.

\section{Figure 1}

Schematic diagrams of nerve root sedimentation sign. A, negative sedimentation sign; B, positive sedimentation sign; $C$, the diagram of negative nerve root sedimentation sign in MRI. The nerve roots were settled to the dorsal side of the dural sac. 


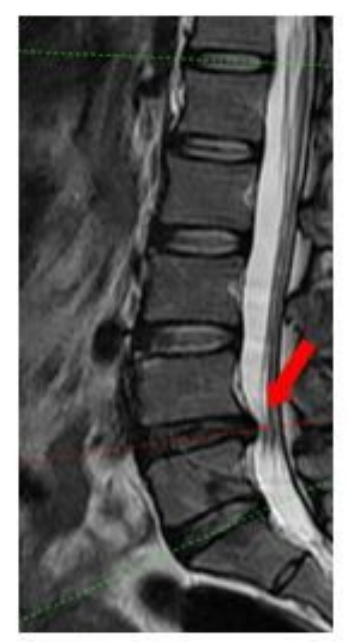

a the sagittal image of L4/5 lumbar disc hemiation in MRI with T2-weighted

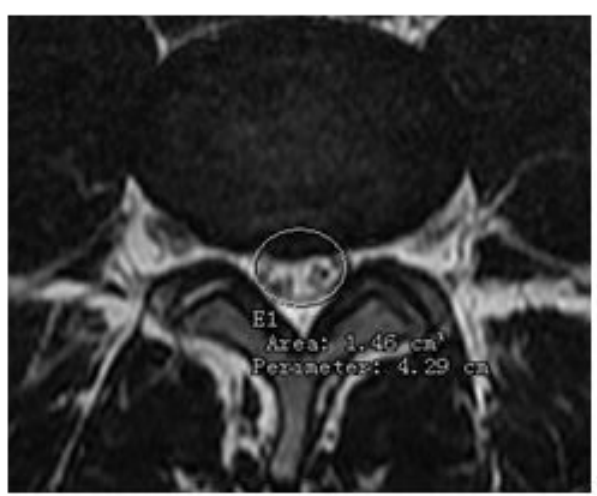

b the cross-sectional area of dural $\operatorname{sac}\left(\mathrm{S}_{1}\right)$

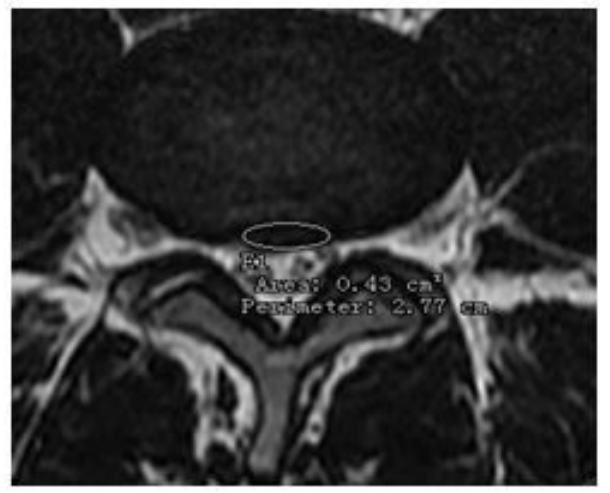

c the cross-sectional area of the hemiated $\operatorname{disc}\left(\mathrm{S}_{2}\right)$

\section{Figure 2}

The ratio of compressed area of dural sac (S2/S1). A, the sagittal image of $L 4 / 5$ lumbar disc herniation in MRI with T2-weighted; B, the cross-sectional area of dural sac(S1); C, the cross-sectional area of the herniated disc(S2). 